\title{
Prevalence and Determinants of Pre-lacteal Feeding: Insights from the 2017 Indonesia Demographic and Health Survey
}

\author{
Siti Nurokhmah ${ }^{*}$, Siti Masitoh², Kusuma Estu Werdani ${ }^{3}$
}

\begin{abstract}
${ }^{1}$ Department of Nutrition Science, Faculty of Health Science, Universitas Muhammadiyah Surakarta, Surakarta, Indonesia
${ }^{2}$ National Institute of Health Research and Development, Indonesian Ministry of Health, Central Jakarta, Indonesia

${ }^{3}$ Department of Public Health, Faculty of Health Sciences, Universitas Muhammadiyah Surakarta, Surakarta, Indonesia
\end{abstract}

\begin{abstract}
Pre-lacteal feeding is widely known as a distraction to exclusive breastfeeding, and the malpractice continues to be prevalent in Indonesia. Therefore, this study aimed to explore the potential determinants of pre-lacteal feeding among mothers of infants below 24 months. A sample of 6,455 mother-infant pairs from the 2017 Indonesia Demographic and Health Survey (IDHS) was used. Also, multivariate logistic regression was employed to identify factors associated with pre-lacteal feeding practice. In Indonesia, $44 \%$ of infants were introduced to solid/liquid feeds in their first three days of life. Infant formula was the most common pre-lacteal feed given, followed by any other milk, plain and sugar water, and honey. Early initiation of breastfeeding and living in an urban area was protective method against pre-lacteal feeding (AOR: $0.24 ; 95 \% \mathrm{Cl}: 0.21-0.28 ; \mathrm{AOR}: 0.76 ; 95 \% \mathrm{Cl}: 0.65-0.90$, respectively), while cesarean delivery acted as a risk factor (AOR: 1.36; 95\% Cl: 1.14-1.63). Meanwhile, gender role attitude, parity, perceived birth size, and household wealth index was also associated with pre-lacteal feeding. Overall, the percentage of mothers introducing pre-lacteal feeds was still high. The modifiable covariates associated with pre-lacteal feedings, such as early initiation of breastfeeding, parity, and birth size, were the major factors discouraging this practice.
\end{abstract}

Keywords: breastfeeding, determinants, early initiation of breastfeeding, Indonesia, pre-lacteal feeding

\section{Introduction}

Breastfeeding has a wide range of benefits for infants throughout their life cycle. ${ }^{1}$ It protects from illnesses or infections, contributing to the reduction of morbidity and mortality. ${ }^{2}$ Besides the short-term benefits, this practice is also associated with a lower risk of several diseases, such as type 2 diabetes, leukemia, celiac, atopic, and inflammatory bowel diseases. ${ }^{3}$ From an economic standpoint, breastfeeding save costs associated with several illnesses, in addition to the costs of using other substitutes. ${ }^{4}$

Generally, pre-lacteal feeding is defined as introducting any solid or liquid feed besides breastmilk in the first three days of life. This has been proven as one of the barriers towards the recommended breastfeeding practices, as well as other factors, such as delaying initiation, shortening the duration, and disrupting exclusive breastfeeding. ${ }^{5}$ The common pre-lacteal feeds given to infants differ across regions and parts of the world, such as in the Maldives, where honey and dates were parts of ritual food given in the first three days of life. Meanwhile, in Ethiopia, raw butter, plain water, and

Correspondence*: Siti Nurokhmah, Department of Nutrition Science, Faculty of Health Science, Universitas Muhammadiyah Surakarta, A. Yani Street, Tromol Pos I Pabelan, Surakarta 16424, Indonesia, Email: siti.nurokhmah@ums.ac.id, Phone: +62 271717417 milk (besides breastmilk) were among the most commonly found pre-lacteal feeds. ${ }^{6,7}$ In Indonesia, most mothers, preferred infant formula, which is similar in South Africa, Bangladesh, and Nepal.8-11

Pre-lacteal feeding is practiced in many regions, even where the breastfeeding rate is high, such as Uganda and Indonesia. In both countries, breastfeeding and given a substitute feed in the first three days of life are believed to be norms. ${ }^{12}$ A study also found that for almost all women, introducing pre-lacteal feeding is not based on health or nutritional concerns. However, it is determined by cultural beliefs or family influence. ${ }^{13}$ Infants given pre-lacteal feeding are not exclusively breastfed by definition. Therefore, encouragements on the avoidance of this practice are crucial for improvement. Also, information about the factors associated with it needs to be obtained. Studies on the determinants of pre-lacteal feeding are found in this literature, while for the Indonesian context, this topic has not been extensively explored. This study aimed to identify factors associated with pre-lacteal feeding among infants under two years of age using the data from the Indonesia Demographic 
and Health Survey (IDHS) 2017.

\section{Method}

This study employed the Indonesia Demographic and Health Survey (IDHS) data conducted in 2017.14 A total of 6,455 mother-infant pairs were included in this assay. At the same time, the data used for the analysis of prelacteal feeding practice were restricted to the last birth in the past two years. A full explanation of the methodology, design, and sampling of the IDHS was found in previous studies. ${ }^{14}$ Furthermore, the sampling frame used the 2010 population census data, which was updated specifically for the selected blocks (clusters). The design used a two-stage stratified sampling. Several clusters were selected from 1,970 in 34 provinces, with systematic selection proportional to the size (size: the number of households) in stage one. Then, in stage two, 25 households were selected systematically. Questionnaires - that was the source of the dataset used in this paper-were administered to women aged 15-19 years. The information collected included data on family planning, HIV/AIDS, maternal and child health, in which the latter encompass the data on infant feeding practices, as the main topic of this analysis.

Pre-lacteal feeding, which is defined as the practice of introducing any liquid or solid feed, except breast milk in the first three days after delivery, was the dependent variable. The independent covariates included the infant, maternal, and household characteristics based on the conceptual framework for breastfeeding, which was adapted from previous publications. ${ }^{1,15}$ Infant characteristics included sex (male and female), age in months (0-5, 6-11, 12-23), perceived birth size (smaller-thanaverage, average, larger-than-average), and birth type (single and multiple). Maternal factors included age in years $(<18,18-34,>34)$, education (primary/no formal education, secondary/post-secondary), occupation (employed and unemployed), parity or the number of children born $(\leq 2,>2)$, place of delivery (home or health facility), birth attendant (health professional and traditional birth attendant), and type of delivery (noncesarean and cesarean). Variables related to women empowerment, such as decisions on health spending (respondent alone, none, or others), and gender role attitudes ('conforming' or 'non-conforming') were also included. Respondents who agreed with at least one reason for wife-beating were categorized as 'conforming' because they complied with the husband's decision or society's norms. While those that did not agree with all reasons for wife-beating were included in the 'nonconforming' category. There were three household-level variables included in the analysis, namely residence (urban or rural), wealth quintile, and household size $(\leq 5$, $>5)$.
All statistical analysis were performed using Stata 15.1 (StataCorp, College Station, Texas, USA-license of the software is available), by taking into account the study design of cluster survey and sample weights. Descriptive statistics were presented in proportions. Analysis of pre-lacteal feeding determinants was conducted using a logistic regression model, where the final model was determined through a backward logistic regression with a significance of $p$-value $<0.05$. The second step was to run the model while excluding covariates with the highest $\mathrm{p}$-value in the full design, then the changes were observed. When the exclusion of this variable did not change the odds ratio(s) by at least $10 \%$, it is left out forever. This second step was repeated until the final model containing independent variables with pvalues less than 0.05 fitted in.

\section{Results}

More than two-thirds of the respondents were at the age of 20-35 years, and almost 25\% of them only attended primary schools or no formal education (Table 2 ). The proportion of not working mothers was $3.4 \%$ higher than those employed in any sector. The percentage of infants introduced to pre-lacteal feed was $44.0 \%$ and almost equally distributed in all age groups (Table 1 ). More than half of mothers, or $24.7 \%$ of all respondents chose the infant formula followed by any milk, plain and sugar water, and honey when asked about what type of pre-lacteal feed preferred.

The majority of the respondents had one or two children, and $18.8 \%$ of respondents underwent cesarean section for their last child. More than $80 \%$ of women delivered in a health facility and had health professionals as their birth attendants. There were $88.9 \%$ of infants which birth sizes were perceived as average or larger by their mothers. Table 2 also shows that almost $70 \%$ of the respondents agreed with wife-beating. As for health spending decisions, $44.9 \%$ of women decided it by themselves. Early initiation of breastfeeding was practiced by almost $60 \%$ of respondents. Both the place of residence and the wealth index distribution were almost equally distributed in each category. More respondents were living in a smaller household with five or fewer family members.

Table 1. Distribution of Pre-lacteal Feeding Practice by Infant Age

\begin{tabular}{lccc}
\hline & & \multicolumn{2}{c}{ Given Pre-lacteal Feeds } \\
\cline { 3 - 4 } Age of Infant (Months) & $\mathbf{N}$ & $\mathbf{n}$ & $\%$ \\
\hline $0-5$ & & 737 & 45.0 \\
$6-11$ & 1,616 & 693 & 42.8 \\
$12-23$ & 1,597 & 1,486 & 44.1 \\
\hline $0-23$ & 3,242 & 2,916 & 44.0 \\
\hline
\end{tabular}


Table 2. Respondents' Characteristics of Pre-lacteal Feeding Practice

\begin{tabular}{|c|c|c|c|}
\hline Variable & Category & $\mathbf{N}$ & $\%$ \\
\hline \multicolumn{4}{|l|}{ Infant characteristic } \\
\hline \multirow{2}{*}{ Infant sex } & Male & 3,344 & 51.4 \\
\hline & Female & 3,111 & 48.6 \\
\hline \multirow[t]{2}{*}{ Birth type } & Single & 6,416 & 99.4 \\
\hline & Multiple & 39 & 0.6 \\
\hline \multirow[t]{3}{*}{ Perceived birth size } & Small & 801 & 11.1 \\
\hline & Average & 3,460 & 57.7 \\
\hline & Large & 2,104 & 31.2 \\
\hline \multicolumn{4}{|l|}{ Maternal characteristic } \\
\hline \multirow{3}{*}{ Maternal age (years) } & $<20$ & 309 & 4.4 \\
\hline & $20-35$ & 4,950 & 76.8 \\
\hline & $\geq 35$ & 1,196 & 18.8 \\
\hline \multirow[t]{3}{*}{ Maternal education } & College or higher & 1,270 & 16.9 \\
\hline & Secondary & 3,657 & 58.9 \\
\hline & Primary/no formal education & 1,528 & 24.2 \\
\hline \multirow[t]{2}{*}{ Occupation } & Not working & 3,511 & 56.7 \\
\hline & Working & 2,938 & 43.3 \\
\hline \multirow[t]{2}{*}{ Parity } & $\leq 2$ & 4,159 & 68.3 \\
\hline & $>2$ & 2,296 & 31.7 \\
\hline \multirow[t]{2}{*}{ Antenatal care visits (times) } & $\geq 4$ & 5,700 & 90.8 \\
\hline & $<4$ & 731 & 9.2 \\
\hline \multirow[t]{2}{*}{ Type of delivery } & Non-caesarean & 5,281 & 81.2 \\
\hline & Caesarean & 1,170 & 18.8 \\
\hline \multirow{2}{*}{ Birth attendants } & Health professional & 5,895 & 93.0 \\
\hline & Non-health professional & 558 & 7.0 \\
\hline \multirow[t]{2}{*}{ Place of delivery } & Health facility & 5,057 & 83.5 \\
\hline & Non-health facility & 1,397 & 16.5 \\
\hline \multirow[t]{2}{*}{ Gender role attitude } & Conforming & 4,088 & 69.0 \\
\hline & Non-conforming & 2,171 & 31.0 \\
\hline \multirow[t]{2}{*}{ A decision on health spending } & Respondent alone & 2,747 & 44.9 \\
\hline & Not respondent alone or other & 3,565 & 55.1 \\
\hline \multirow[t]{2}{*}{ Early initiation of breastfeeding } & Yes & 3,732 & 59.5 \\
\hline & No & 2,723 & 40.5 \\
\hline \multicolumn{4}{|l|}{ Household characteristics } \\
\hline \multirow[t]{5}{*}{ Wealth index } & Poorest & 1,747 & 20.2 \\
\hline & Poor & 1,285 & 20.2 \\
\hline & Middle & 1,172 & 19.8 \\
\hline & Rich & 1,161 & 20.8 \\
\hline & Richest & 1,090 & 19.0 \\
\hline \multirow[t]{2}{*}{ Place of residence } & Urban & 3,145 & 48.3 \\
\hline & Rural & 3,310 & 51.7 \\
\hline \multirow[t]{2}{*}{ Household size } & $\leq 5$ & 3,531 & 60.0 \\
\hline & $>5$ & 2,924 & 40.0 \\
\hline
\end{tabular}

Table 3 shows the bivariate analysis results in which early initiation and type of delivery were strongly associated with pre-lacteal feeding ( $\mathrm{p}$-value $<0.0001$ ). Cesarean delivery almost doubled the odds of pre-lacteal feeding $(\mathrm{OR}=1.82,95 \% \mathrm{CI}=1.55-2.15)$. However, infants with no early breastfeeding initiation had around four times higher odds of given pre-lacteal feeds $(\mathrm{OR}=$ $4.37,95 \% \mathrm{CI}=3.81-5.01)$. Other variables showing association with pre-lacteal feeding in this unadjusted analysis were parity, wealth index, and place of residence.

Results of the multivariate logistic regression were presented in Table 4. In the initial model (results not shown), there were nine independent covariates were included as the p-value in the bivariate analysis (Table 3 ), which was less than 0.25 . In the end, two variables were excluded, which resulted in only seven covariates kept in the final model. Early initiation of breastfeeding and type of delivery remained the strongest predictors. Then, the adjustment for other variables only changed the odds ratios at around $8 \%$ for early initiation of breastfeeding and $25 \%$ for the type of delivery with no change in their p-values. Respondents with more than two children were less in introducing pre-lacteal feeds (AOR $=0.83,95 \% \mathrm{CI}=0.71-0.96)$. Mothers who perceived that their infant was smaller than average had $1.43(95 \% \mathrm{CI}=1.15-1.79)$ times the odds of introducing pre-lacteal feeding. Living in rural areas was associated with the higher practice of pre-lacteal feeding, increasing the odds by $31 \%$. Gender role attitudes and wealth index were also found to be associated with this practice.

\section{Discussion}

This study found that almost half of the mothers introduced pre-lacteal feeds, and the infant formula was the most common feed given. Analysis of the determinants of pre-lacteal feeding showed that perceived birth size, type of delivery, parity, early initiation of breastfeeding, gender role attitude, household wealth index, and place of residence were associated with prelacteal feeding. Not all variables that were initially assumed to be correlated with the result showed evidence of association. Variables reported to be predictors of prelacteal feeding in previous reports were maternal education, employment status, antenatal care visit, and place of birth. However, these variables showed no correlation in this study. ${ }^{16,17}$

Pre-lacteal feeding was practiced by $44 \%$ of mothers, whose ages were less than 24 months in 2017. This figure was lower than that of previous surveys, which was $60 \%$ in 2012 and $65 \%$ in $2007 .{ }^{14}$ Compared to other ASEAN countries, pre-lacteal feeding in Indonesia was lower than Vietnam $(73.3 \%)$ and higher than Laos (35\%). ${ }^{18,19}$ Some countries, such as Nepal and Ethiopia have lower figures with $29 \%$ and $8 \% .20,21$ Differences in geographical characteristics, exposure to modern lifestyle, and policies regarding breastfeeding might be the reasons for these differences.

Pre-lacteal feeding is associated with early initiation and exclusive breastfeeding. Infants with delayed breastfeeding initiation are more likely to receive the prelacteal feed. Introducing pre-lacteal feeding is negatively associated with the exclusive type as well.22 The downward trend of pre-lacteal feeding in the last decade was followed by the increasing prevalence of early initiation or exclusive breastfeeding. ${ }^{14,23}$ However, there were still more than half of the children not exclusively breastfeed. Therefore, more effort to improve the exclusive type or infant feeding practices should be prioritized.

Observing the pre-lacteal feeds given, infant formula 
Table 3. Bivariate Analysis for the Determinants of Pre-lacteal Feeding

\begin{tabular}{|c|c|c|c|c|c|c|}
\hline \multirow{2}{*}{ Variable } & \multirow{2}{*}{ Category } & \multicolumn{2}{|c|}{ Pre-lacteal Feeding } & \multirow{2}{*}{ OR } & \multirow{2}{*}{ 95\% CI } & \multirow{2}{*}{ p-value } \\
\hline & & Yes & No & & & \\
\hline \multirow[t]{3}{*}{ Infants age (months) } & $0-5$ & $737(54.0 \%)$ & $879(55.0 \%)$ & 1 & & \\
\hline & $6-11$ & $693(42.8 \%)$ & $904(57.2 \%)$ & 0.91 & $0.77-1.09$ & 0.32 \\
\hline & $12-23$ & $1,486(44.0 \%)$ & $1,756(56.0 \%)$ & 0.96 & $0.83-1.12$ & 0.63 \\
\hline \multirow[t]{2}{*}{ Infants sex } & Male & $1,513(43.8 \%)$ & $1,831(56.2 \%)$ & 1 & & \\
\hline & Female & $1,403(44.1 \%)$ & $1,708(55.9 \%)$ & 1.01 & $0.89-1.15$ & 0.82 \\
\hline \multirow[t]{2}{*}{ Birth type } & Single & $2,892(43.9 \%)$ & $3,524(56.1 \%)$ & 1 & & \\
\hline & Multiple & $24(50.7 \%)$ & $15(49.1 \%)$ & 1.31 & $0.59-2.90$ & 0.5 \\
\hline \multirow[t]{3}{*}{ Perceived birth size } & Small & $415(53.3 \%)$ & $386(46.7 \%)$ & 1.57 & $1.30-1.91$ & $<0.001$ \\
\hline & Average & $1,529(42.0 \%)$ & $1,931(58.0 \%)$ & 1 & & \\
\hline & Large & $935(44.2 \%)$ & $1,169(55.8 \%)$ & 1.09 & $0.95-1.26$ & 0.21 \\
\hline \multirow[t]{2}{*}{ Maternal age } & Not at risk & $2,203(44.2 \%)$ & $2,687(55.8 \%)$ & 1 & & \\
\hline & At risk & $713(43.3 \%)$ & $852(56.7 \%)$ & 0.97 & $0.84-1.11$ & 0.63 \\
\hline \multirow[t]{3}{*}{ Maternal education } & College or higher & $624(45.9 \%)$ & $646(54.1 \%)$ & 1 & & \\
\hline & Secondary & $1,650(44.3 \%)$ & $2,007(55.7 \%)$ & 0.94 & $0.79-1.11$ & 0.46 \\
\hline & Primary or no formal education & $642(41.7 \%)$ & $886(58.3 \%)$ & 0.84 & $0.69-1.03$ & 0.1 \\
\hline \multirow[t]{2}{*}{ Occupation } & No & $1,582(43.8 \%)$ & $1,929(56.2 \%)$ & 1 & & \\
\hline & Yes & $1,332(44.2 \%)$ & $1,606(55.8 \%)$ & 1.01 & $0.90-1.15$ & 0.8 \\
\hline \multirow[t]{2}{*}{ Parity } & $\leq 2$ & $1,960(45.8 \%)$ & $2,199(54.2 \%)$ & 1 & & \\
\hline & $>2$ & $956(40.0 \%)$ & $1,340(60.0 \%)$ & 0.79 & $0.69-0.90$ & 0.001 \\
\hline \multirow[t]{2}{*}{ Antenatal care visits } & $\geq 4$ & $2,584(43.9 \%)$ & $3,116(56.1 \%)$ & 1 & & \\
\hline & $<4$ & $322(44.2 \%)$ & $409(55.8 \%)$ & 1.01 & 0.821 .25 & 0.92 \\
\hline \multirow[t]{2}{*}{ Type of delivery } & Non-caesarean & $2,215(41.2 \%)$ & $3,066(58.8 \%)$ & 1 & & \\
\hline & Caesarean & $700(56.1 \%)$ & $470(43.9 \%)$ & 1.82 & $1.55-2.15$ & $<0.001$ \\
\hline \multirow[t]{2}{*}{ Birth attendant } & Health professional & $2,689(44.2 \%)$ & $3,206(55.8 \%)$ & 1 & & \\
\hline & Non-health professional & $226(40.1 \%)$ & $332(59.9 \%)$ & 0.84 & $0.63-1.12$ & 0.25 \\
\hline \multirow[t]{2}{*}{ Place of delivery } & Health facility & $2,296(43.7 \%)$ & $2,761(56.2 \%)$ & 1 & & \\
\hline & Non-health facility & $620(45.0 \%)$ & $777(55.0 \%)$ & 1.05 & $0.89-1.25$ & 0.55 \\
\hline \multirow[t]{2}{*}{ Gender role attitude } & Conforming & $1,802(43.2 \%)$ & $2,284(56.8 \%)$ & 1 & & \\
\hline & Non-conforming & $1,017(45.7 \%)$ & $1,154(54.3 \%)$ & 1.11 & $0.97-1.27$ & 0.13 \\
\hline \multirow[t]{2}{*}{ Decision on health spending } & Respondent alone & $1,222(43.2 \%)$ & $1,525(56.8 \%)$ & 1 & & \\
\hline & Not respondent alone or other & $1,632(44.8 \%)$ & $1,933(55.2 \%)$ & 1.06 & $0.94-1.21$ & 0.33 \\
\hline \multirow[t]{2}{*}{ Early initiation of breastfeeding } & Yes & $1,153(29.7 \%)$ & $2,579(70.3 \%)$ & 1 & & \\
\hline & No & $1,763(64.8 \%)$ & $960(35.1 \%)$ & 4.37 & $3.81-5.01$ & $<0.001$ \\
\hline \multirow[t]{5}{*}{ Wealth index } & Poorest & $701(40.3 \%)$ & $1,046(59.7 \%)$ & 0.8 & $0.65-0.98$ & 0.03 \\
\hline & Poor & $609(44.4 \%)$ & $676(55.6 \%)$ & 0.94 & $0.77-1.15$ & 0.56 \\
\hline & Middle & $553(45.9 \%)$ & $619(54.4 \%)$ & 1 & & \\
\hline & Rich & $557(46.6 \%)$ & $604(53.4 \%)$ & 1.03 & $0.84-1.25$ & 0.77 \\
\hline & Richest & $496(42.5 \%)$ & $594(57.5 \%)$ & 0.87 & $0.70-1.08$ & 0.2 \\
\hline \multirow[t]{2}{*}{ Place of residence } & Urban & $1,405(41.7 \%)$ & $1,740(58.3 \%)$ & 1 & & \\
\hline & Rural & $1,511(46.1 \%)$ & $1,799(56.0 \%)$ & 1.19 & $1.02-1.37$ & 0.01 \\
\hline \multirow[t]{2}{*}{ Household size } & $\leq 5$ & $1,623(44.1 \%)$ & $1,908(55.9 \%)$ & 1 & & \\
\hline & $>5$ & $1,293(43.8 \%)$ & $1,631(56.2 \%)$ & 0.99 & $0.87-1.12$ & 0.85 \\
\hline
\end{tabular}

Notes: $\mathrm{OR}=$ Odd Ratio, $\mathrm{CI}=$ Confidence Interval

was the most prevalent choice, as also shown in previously conducted IDHS and another national survey, namely Basic Health Surveys (Riskesdas). ${ }^{8}$ Although the overall percentage of pre-lacteal feeding decreased over time, proportions of infant formula feeding in the first three days of life saw an upward trend. 8 Massive promotions of infant formula contributed to this condition. In Vietnam, infant formula was also the most popular pre-lacteal feed chosen. ${ }^{13,18}$

This study added the literature on factors associated with the pre-lacteal feeding practice in the Indonesian context. The only infant level characteristic showed an association between pre-lacteal feeding and perceived birth size: mothers perceiving their newborns were smaller than average, to be given pre-lacteal feeds. This independent covariate acted as a strong predictor for prelacteal feeding either in bivariate or multivariate analysis. Previous studies in South Asia using DHS data also showed similar conclusions regarding this variable. ${ }^{15}$ However, a meta-analysis on Ethiopian studies did not include birth size among their determinants. ${ }^{17}$ Small size newborns often have difficulties when breastfeeding, normally due to problems with their ability to suck the breastmilk. This problem causes frustration for the infants, making them end up crying. Mothers often interpret infant crying as hunger and think of it that the breastmilk alone was not enough, therefore, their newborns should be given food/liquid besides breastmilk. 
Table 4. The Final Model for the Determinants of Pre-lacteal Feeding in Indonesia

\begin{tabular}{llrrr}
\hline Variable & Category & AOR & 95\% CI & p-value \\
\hline Perceived birth size & Smaller than average & 1.43 & $1.15-1.79$ & 0.001 \\
& Average & 1 & 1 & \\
& Bigger than average & 1.08 & $0.92-1.26$ & 0.35 \\
Type of delivery & Non-caesarean & 1 & 1 & \\
& Caesarean & 1.36 & $1.14-1.63$ & 0.001 \\
Parity & $\leq 2$ & 1 & 1 & \\
Early initiation of breastfeeding & Yes & 0.83 & $0.71-0.96$ & 0.01 \\
& No & 1 & 1 & \\
Gender role attitude & Conforming & 4.13 & $3.58-4.75$ & $<0.001$ \\
& Nonconforming & 1 & 1 & \\
Wealth index & Poorest & 0.72 & $1.00-1.32$ & 0.05 \\
& Poor & 0.90 & $0.72-1.91$ & 0.005 \\
& Middle & 1 & 1 & 0.36 \\
Rich & 0.97 & $0.79-1.20$ & 0.81 \\
Place of residence & Richest & 0.90 & $0.70-1.14$ & 0.38 \\
& Urban & 1 & 1 & \\
& Rural & 1.31 & $1.11-1.54$ & 0.001 \\
\hline
\end{tabular}

Notes: $\mathrm{AOR}=$ Adjusted Odd Ratio, $\mathrm{CI}=$ Confidence Interval

Mothers delivering small-size infants also think that given pre-lacteal feeds help their newborns to gain weight faster.

Cesarean delivery was consistently reported as a factor discouraging recommended breastfeeding practices in developing countries. ${ }^{13,24}$ This study also had the same conclusion, in which mothers with cesarean delivery had around 50\% higher likelihood to introduce pre-lacteal feeding. Mothers delivered by c-section, either as an emergency or planned c-section, were reported to have difficulties with breastfeeding due to the inability to produce enough milk, inverted or flat nipples, and feeling of discomfort due to swollen or painful breasts, and soreness nipples. ${ }^{25}$ These conditions drive mothers to introduce pre-lacteal feeding, and this chance was higher when there was no assistance or lactation support available. Besides, women undergoing c-sections were less likely to plan to breastfeed compared to those with vaginal deliveries. ${ }^{25}$

Women with higher parity had a lower likelihood to introduce pre-lacteal feeding. ${ }^{13}$ In Asia, first-time mothers were more likely to give pre-lacteal feeds. They had a lower chance to adopt other recommended infant feeding practices, such as early initiation and exclusive breastfeeding, and appropriate complementary feeding. ${ }^{13,26,27}$ A possible explanation for this result was that women with higher parity have more experience in dealing with infant feeding. They learned what they had done for their previous children, what worked, and those that did not.

Compared to other covariates in this analysis, early initiation of breastfeeding was not only the most solid predictor. However, it also had the biggest effect size. This result was in line with existing literature stating that early initiation acts as protective toward pre-lacteal feeding. ${ }^{17}$ The period between delivery and late breastfeeding might increase the probability of mothers that gives pre-lacteal feed. Meanwhile, advice from family members might influence mothers' decisions, whether to give pre-lacteal feed or not. A study in southern Ethiopia showed that grandparents were the most common external source of information to introduce pre-lacteal feeding. ${ }^{5}$

Findings on women empowerment-related variables (gender role attitude) showed that the factor of 'nonconforming', women, predict pre-lacteal feeding. This finding was similar to that of the analysis on Afghanistan population surveys, which showed that women empowerment was associated with the introduction of pre-lacteal feeding. ${ }^{15}$ In the latter study, another variable, namely women's decision-making autonomy, also predicted that autonomous women were more likely to give pre-lacteal feed. This variable was not included in the present study; however, a variable representing decision on health spending was analyzed and showed that health spending was not associated with pre-lacteal feeding.

Analysis showed some evidence of the association between socioeconomic variables, which were wealth index and pre-lacteal feeding. However, this correlation was only significant in the poorest group. Mothers from the lowest wealth-quintile were less likely to introduce pre-lacteal feeds than those from the middle. Studies in Vietnam and Laos also found similar results that a high socioeconomic status was a risk factor for pre-lacteal feeding. ${ }^{19}$ A possible explanation for this finding is because breastmilk substitutes, such as infant formula, were highly regarded and perceived as a symbol of a 
modern lifestyle. Women of higher socioeconomic status were able to afford it, unlike the underprivileged. A similar finding was also reported on the association between breastfeeding and socioeconomic factors that, breastfeeding was more common in lower socioeconomic women. ${ }^{2}$

Living in rural areas increased the odds of introducing pre-lacteal feeding, as shown in this analysis, was also reported by several studies on African populations. ${ }^{17,28}$ Cultural beliefs and local norms are more commonly practiced by rural communities. The lack of access to information related to optimal infant feeding practices was among the reasons behind the higher proportion of rural women introducing pre-lacteal feeding. Besides, variables related to cultural practices and information about Infant and Young Child Feeding (IYCF) were not included in this study.

This study used a nationally representative dataset on a large scale which provides reliable results for the analysis. The variables also used international definitions to be compared with previous studies. Analysis conducted in this report also took into account a complex survey design. Some limitations were unavoidable, while data on pre-lacteal feeding were obtained using the recall method, resulting in memory bias as respondents were asked, what happened in 0-22 months before the interview. Secondly, analyzing data from a crosssectional design, reflected the association between variables, while causal inference was restricted. There was also one variable with several missing values that were not excluded in the multivariate analysis due to its very strong association with the results. However, this potential bias caused by missing data should not be significant since the data missing did not differ between clusters.

\section{Conclusion}

Perceived birth size, type of delivery, parity, early initiation of breastfeeding, and place of residence were found to strongly associate with pre-lacteal feeding. Infants born with smaller-size than average, such as those delivered by cesarean section, are more likely to receive a pre-lacteal feed. Mothers with one or two children have higher odds of introducing and engaging in this practice. By contrast, respondents that initiated breastfeeding within the first hour after delivery have lower odds of giving a pre-lacteal feed. In contrast, urban women have a higher likelihood of giving this feed. Household wealth index and gender role attitude showed some evidence of association with pre-lacteal feeding. The poorest respondents had a higher risk of introducing solid/ liquid feeds in the first three days after delivery. The 'nonconforming women' were more likely to engage in this practice. Therefore, interventions are needed to discourage mothers from introducing pre-lacteal feeding.

Efforts should also be made by directly targeting modifiable covariates, such as early initiation of breastfeeding and specific measures on cesarean delivery. Health facilities need to ensure that early initiation of breastfeeding is part of the procedure either for normal or cesarean delivery. This was also found in previous studies which showed that early initiation of breastfeeding rate was lower in women with cesarean compared to those with normal delivery. Health staffs also need sufficient skills and knowledge to educate women and their families about the recommended infant feeding practices. This effort is effective when the procedures in the health facilities include this activity. Further studies explaining the associations between early initiation of breastfeeding, cesarean delivery, and prelacteal feeding are needed to formulate more effective interventions.

Women with more than two children were chosen to be part of the community-based interventions related to infant feeding and targeting pregnant women as they already have experience dealing with this practice. The analysis also showed that they were less likely to introduce pre-lacteal feeding. Moreover, it is important to address this issue, as the smaller-size-born infants are more prone to receive pre-lacteal feeds, and the education from health staff on how to prevent low-birthweight is also crucial. Special attention should be given to women from the lowest socioeconomic background and those with non-conforming gender role attitudes, as they have a higher chance of giving pre-lacteal feeds.

In Indonesia, all these efforts should be integrated into community health centers with more massive education and counseling, targeting the pregnant women and the family. In delivery, health facilities play a crucial role in counseling and education on infant feeding, and it is to support all breastfeeding-friendly policies.

\begin{abstract}
Abbreviations
AOR: Adjusted Odds Ratio; CI: Confidence Interval; IDHS: Indonesia Demographic and Health Survey; HIV/AIDS: Human Immunodeficiency Virus/Acquired Immune Deficiency Syndrome; OR: Odds Ratio; ASEAN: The Association of Southeast Asian Nations; AOR: Adjusted Odds Ratio; CI: Confidence Interval; DHS: Demographic and Health Survey; IYCF: Infant and Young Child Feeding.
\end{abstract}

\section{Ethics Approval and Consent to Participate}

The ethics approval of the 2017 IDHS was received from ICF Macro Institutional Review Board (IRB), Maryland, USA. This study was a secondary data analysis of publicly accessible data. Informed consent was obtained from all participants.

Competing Interest 
The author declares that there are no significant competing financial, professional, or personal interests that might have affected the performance or presentation of the work described in this manuscript.

\section{Availability of Data and Materials}

The materials and dataset used in this study were accessed at https://dhsprogram.com/data/available-datasets.cfm.

\section{Authors' Contribution}

SN was responsible for the overall concept of the study, analysis, and writing the manuscript. SM helped the concept and statistical analysis, while KEW contributed to the writing. All authors discussed the content of this manuscript and approved the final version.

\section{Acknowledgment}

The authors would like to acknowledge Indonesia Demographic and Health Survey (IDHS) and ICF Macro Institutional Review Board for permission to use the dataset collected in 2017. Sincere gratitude to Dr. Aurélie Bechoff for proofreading this report.

\section{References}

1. Victora CG, Bahl R, Barros AJD, França GVA, Horton S, Krasevec J, et al. Breastfeeding in the 21st century: epidemiology, mechanisms, and lifelong effect. Lancet. 2016; 387 (10017): 475-90.

2. Rollins NC, Bhandari N, Hajeebhoy N, Horton S, Lutter CK, Martines JC, et al. Why invest, and what it will take to improve breastfeeding practices?. Lancet. 2016; 387 (10017): 491-504.

3. Brahm P, Valdés $V$. The benefits of breastfeeding and risks associated with not breastfeeding. Revista Chilena de Pediatría. 2017; 88 (1): 714.

4. Walters D, Horton S, Siregar AYM, Pitriyan P, Hajeebhoy N, Mathisen R, et al. The cost of not breastfeeding in Southeast Asia. Health Policy and Planning. 2016; 31 (8): 1107-16.

5. Amele EA, Demissie BW, Desta KW, Woldemariam EB. Prelacteal feeding practice and its associated factors among mothers of children age less than 24 months old in Southern Ethiopia. Italian Journal of Pediatrics. 2019; 45 (1): 15.

6. Belachew AB, Kahsay AB, Abebe YG. Individual and community-level factors associated with introduction of prelacteal feeding in Ethiopia. Archives of Public Health. 2016; 74 (1): 1-11.

7. Raheem RA, Binns CW, Chih HJ, Sauer K. Determinants of the introduction of prelacteal feeds in the Maldives. Breastfeeding Medicine. 2014; 9 (9): 473-8.

8. Kementerian Kesehatan Republik Indonesia. Laporan nasional riset kesehatan dasar 2018. 2018. p. 1-582.

9. Doherty T, Sanders D, Jackson D, Swanevelder S, Lombard C, Zembe W, et al. Early cessation of breastfeeding amongst women in South Africa: an area needing urgent attention to improve child health. BMC Pediatrics. 2012; 12: 105.

10. Karkee R, Lee AH, Khanal V, Binns CW. Initiation of breastfeeding and factors associated with prelacteal feeds in Central Nepal. Journal of Human Lactation. 2014; 30 (3): 353-7.

11. Ara G, Khanam M, Papri N, Nahar B, Haque MA, Kabir I, et al. Peer counselling improves breastfeeding practices: a cluster randomized controlled trial in urban Bangladesh. Maternal \& Child Nutrition. 2018; 14 (3): e12605.

12. Flax VL, Hawley I, Ryan J, Chitukuta M, Mathebula F, Nakalega R, et al. After their wives have delivered, a lot of men like going out: perceptions of HIV transmission risk and support for HIV prevention methods during breastfeeding in sub-Saharan Africa. Maternal \& Child Nutrition; 2020.

13. Champeny M, Pries AM, Hou K, Adhikary I, Zehner E, Huffman SL. Predictors of breast milk substitute feeding among newborns in delivery facilities in urban Cambodia and Nepal. Maternal \& Child Nutrition. 2019; 15 (S4): e12754.

14. National Population and Family Planning Board, Indonesia. Indonesia demographic and health survey 2017. Jakarta, Indonesia: BKKBN, BPS, Kemenkes, and ICF; 2018.

15. Benedict RK, Craig HC, Torlesse H, Stoltzfus RJ. Trends and predictors of optimal breastfeeding among children 0-23 months, South Asia: analysis of national survey data. Maternal \& Child Nutrition. 2018; 14 Suppl 4: e12698.

16. Agho KE, Ogeleka P, Ogbo FA, Ezeh OK, Eastwood J, Page A. Trends and predictors of prelacteal feeding practices in Nigeria (2003-2013). Nutrients. 2016; 8 (8).

17. Temesgen H, Negesse A, Woyraw W, Getaneh T, Yigizaw M. Prelacteal feeding and associated factors in Ethiopia: systematic review and meta-analysis. Int Breastfeed J. 2018; 13 (1): 49.

18. Nguyen PH, Keithly SC, Nguyen NT, Nguyen TT, Tran LM, Hajeebhoy N. Prelacteal feeding practices in Vietnam: problems and determinant factors. BMC Public Health. 2013; 13: 1-11.

19. Kounnavong S, Pak-gorstein S, Akkhavong K, Palaniappan U. Key determinants of optimal breastfeeding practices in Laos. Food and Nutrition Sciences. 2013; 04 (10).

20. Ministry of Health, Nepal. New ERA: inner city fund international. Nepal demographic and health survey 2016. Kathmandu, Nepal: MOH/Nepal, New ERA, and ICF; 2017.

21. Central Statistical Agency, Ethiopia; Inner City Fund International. Ethiopia demographic and health survey 2016. Addis Ababa, Ethiopia: CSA and ICF; 2017.

22. Paramashanti BA, Hadi H, Gunawan IMA. Timely initiation of breastfeeding is associated with the practice of exclusive breastfeeding in Indonesia. Asia Pacific Journal of Clinical Nutrition. 2016; 25 (Suppl 1): S52-6.

23. Saputri NS, Spagnoletti BRM, Morgan A, Wilopo SA, Singh A, McPake B, et al. Progress towards reducing sociodemographic disparities in breastfeeding outcomes in Indonesia: a trend analysis from 2002 to 2017. BMC Public Health. 2020; 20 (1): 1-15.

24. Saaka M, Hammond AY. Caesarean section delivery and risk of poor childhood growth. Journal of Nutrition and Metabolism. 2020; 1-12.

25. Hobbs AJ, Mannion CA, Mcdonald SW, Brockway M, Tough SC. The impact of caesarean section on breastfeeding initiation, duration and difficulties in the first four months pospartum. BMC Pregnancy Childbirth. 2016; 16 (90): 1-9.

26. Senarath U, Dibley MJ, Agho KE. Factors associated with nonexclusive breastfeeding in 5 east and southeast Asian countries: a multilevel analysis. Journal of Human Lactation. 2010; 26 (3): 24857. 
Nurokhmah et al, Prevalence and Determinants of Pre-lacteal Feeding

27. Patel A, Banerjee A, Kaletwad A. Factors associated with prelacteal feeding and timely initiation of breastfeeding in hospital-delivered infants in India. Journal of Human Lactation. 2013; 29 (4): 572-8.
28. Berde AS, Ozcebe H. Risk factors for prelacteal feeding in subSaharan Africa: a multilevel analysis of population data from twentytwo countries. Public Health Nutrition. 2017; 20 (11): 1953-62. 